\title{
A Polarization-Independent SPR Fiber Sensor
}

\author{
María-Cruz Navarrete • Natalia Díaz-Herrera • \\ Agustín González-Cano • Óscar Esteban
}

Received: 20 April 2009 / Accepted: 12 October 2009 /Published online: 21 October 2009

(C) Springer Science + Business Media, LLC 2009

\begin{abstract}
The resonance of surface plasma waves in metallic layers is a strongly polarization-dependent phenomenon by the very nature of the physical effect responsible of that resonance. This implies the necessity of polarization-controlling elements to be added to any operative surface-plasmon-resonance-based sensor. A fully symmetrical, circular-section double deposition of a metallic and a dielectric layer on a uniform-waist tapered optical fiber (SymDL-UWT) permits us to completely eliminate the dependence on polarization of the plasmon excitation, with the corresponding operative advantages and basic theoretical consequences. We depict the fabrication process of these transducers, which is based on the use of a simple and efficient rotating element developed by us, and show the characteristics of the produced devices. No such device has been depicted up to date. As our experimental results show, this kind of devices can be considered a very good option for the development of simple, compact, and efficient chemical and biological sensors.
\end{abstract}

M.-C. Navarrete

Departamento de Óptica, Facultad de CC. Físicas,

Universidad Complutense,

Ciudad Universitaria $\mathrm{s} / \mathrm{n}$,

28040 Madrid, Spain

N. Díaz-Herrera $(\bowtie) \cdot$ A. González-Cano

Sección departamental de Óptica, Escuela Universitaria de Óptica, Universidad Complutense,

Arcos de Jalón, 118,

28037 Madrid, Spain

e-mail: ndiazher@fis.ucm.es

Ó. Esteban

Departamento de Electrónica, Escuela Politécnica,

Universidad de Alcalá,

28871 Alcalá de Henares, Spain
Keywords Fiber-optic sensors · Surface plasmon resonance $\cdot$ Polarization dependence

\section{SPR and polarization}

As it is well known, in some conditions, a surface plasma wave can be excited in the interface of a metallic and a dielectric media. This wave propagates along the interface, exponentially decaying both in the directions longitudinal and transversal to that surface, and is extremely sensitive to the changes in the optical properties in the media. This property has been very successfully exploited in recent years to develop the surface plasmon resonance (SPR) sensors $[1,2]$. In most of these sensors, the surface plasma wave is excited by an evanescent electromagnetic field produced in a attenuated total reflection in a prism or other refracting element (the so-called Kretchmann configuration). Apart from the conditions related to the angle of incidence or the wavelength of the incident wave, it is critical for the plasmon resonance to provide the right polarization. Surface plasma waves are transverse magnetic (TM)-polarized, and the plasmon resonance phenomenon is very polarization-sensitive [3-5].

This fact implies (although in many depictions of devices in the literature this point is not explicitly expressed) the use of polarization-controlling elements. In principle, when flat layers are used, it is possible to define transverse electric (TE) and TM polarizations with respect to the incidence plane without ambiguity. Then, the light coming out of the source must be polarized, and this polarization must be maintained without fluctuations up to the transducer. This is not always easy, and some schemes have been proposed to decrease the influence of polarization in the measurements of the sensors [6]. But it is the 
very nature of the physical effect of plasma wave excitation which is responsible for this dependence ("polarizationinterrogated" SPR sensors have even been proposed [7]), so we cannot expect to eliminate this dependence or the necessity of polarization-controlling elements for this kind of sensors (with the subsequent increase in the complexity of the setup).

When dealing with fiber-optic sensors based on polished fibers, the situation is similar. SPR fiber-optic sensors are spectrally, not angularly, interrogated, but again the layers deposited on them are flat, and TE and TM directions are well defined. To develop all-fiber systems, in-line polarizers can be used [8]. It is interesting to note that these in-line polarizers are based on the very same phenomenon of plasma wave excitations, and that was in this context that SPR was first explored, SPR polarizers being previous to SPR sensors [9]. But, again, we must assure that, even if one of these elements is used to extinguish TE polarization, the right state of vibration of the field must be maintained until the transducer region. This, at least, implies that the fiber must be kept straight, without torsion and without stress, to avoid birefringence. We have demonstrated an allfiber SPR-based salinity sensor, fully operative for field measurements, and we needed to develop a fairly complex arrangement for the optode to provide a degree of freedom to rotate the in-line polarizer to achieve the right incident polarization [10]. When transforming a working laboratory device to an operative field instrument, this kind of details complicates the setup, and this fact is not explicitly covered in the literature in many cases.

In all the depicted situations, the starting condition for the SPR transducers is to have flat layers. This is natural in the Kretchmann configuration and may be easy from the fabrication point of view in polished-fiber-based sensors. But, at the same time, the existence of well-defined incidence planes maximizes the dependence of the devices with polarization. In the case of fiber-optic sensors, it also complicates theoretical treatment because the cylindrical symmetry of the waveguide is lost when flat layers are deposited on it [11-13]. Tapered optical fibers, which are very convenient devices from many points of view, keep the cylindrical symmetry and can provide access to the evanescent field of the guided modes in a more convenient way [14].

A uniform-waist taper (UWT) is produced by using the so-called traveling-burner setup $[15,16]$, and its fabrication parameters are easily controlled (which is not possible with biconical tapers, made with a fiber splicer [17]). When depositing materials on tapers, we cannot expect to have flat layers any more. This fact has profound implications in the characteristics of the plasma waves excited. The thickness of the layers is no longer constant, and the conditions for the plasmon resonance are fulfilled for different wavelengths, thus producing multiple plasmon dips $[18,19]$. Also, the dependence on polarization is not critical. We still have some dependence, and the use of polarization-controlling elements is adequate to maximize signal, but we can no longer speak of TE and TM polarization since we do not have a well-defined incidence plane. Some authors still use TE and TM to depict the performance of their sensors, but they refer these polarizations arbitrarily to a plane which cannot correspond to the deposited layers [20-22].

A further decrease in polarization dependence can be achieved by covering as much extension of the circular section of the taper as it is possible. A three-step sequential deposition process has been proposed that assures a constancy of the thickness of the deposited layer (only a gold layer is considered) up to an estimated 13\% [20]. This quasicircular deposition is not still, however, fully homogenous, and the dependence on polarization is not completely eliminated.

To provide a fully symmetric deposit, some rotation mechanism must be adapted to the vacuum chamber. Some authors have proposed similar arrangements in different contexts, as we depict in the following paragraph. Also, in some other references, no mention to the deposition process is made, but the symmetry of the deposits is implicitly assumed, which is also very common in those papers dealing with theoretical estimations of SPR sensor performance. However, the technical details of the experimental arrangements are crucial, and the success in the elimination of the polarization dependence relies on them.

What we present here is a fully symmetrical doubly deposited UWT (SymDL-UWT) that shows strictly no dependence with the polarization of the incident light. The introduction of a second dielectric layer has proven to be decisive to control the location of the plasmon resonance, which is very important when moving this resonance outside the usual ranges of refractive indices or wavelengths [23, 24]. We depict the fabrication process, characterize the transducers, and compare its performance in terms of polarization dependence with other devices. We show how polarizationcontrolling elements can be completely eliminated from the experimental setup, which is extremely important when developing field instruments. No device of these characteristics has been presented up to date in the literature, and it is important to note how convenient the introduction of these transducers will be for the development of more compact and robust chemical and biological sensors.

In such an exigent field of applied plasmonics, any improvement of the existing systems can be very relevant for its potential research and commercial implications. The almost paradoxical result of plasmon resonance without polarization dependence is, then, important from this practical point of view, but it has also implications in the theoretical understanding of the dynamics of plasma wave excitation in optical waveguides. 


\section{Fabrication of the devices and experimental setup}

As we have said before, to produce a UWT, we must use a traveling-burner setup. The fiber is heated and, at the same time, gently stretched. The process can be computercontrolled, and the parameters of the taper (waist diameter, waist length, profile of the transition regions) can be selected. This procedure permits us to produce in an easy and fast way highly repeatable geometries, and the tapers can be almost adiabatic [16, 23].

When we introduce the UWTs in the vacuum chamber to deposit the two layers to produce a double-layer uniformwaist tapered (DLUWT) fiber, a characteristic profile of the thickness distribution is produced. We speak of asymmetric deposition (Fig. 1, a), and we have demonstrated that the structures permit multiple plasmon resonances and show a decreased polarization dependence (with respect to that of the devices based on flat layers), these two facts being coherent with the nonconstancy of the thickness of the deposited layers and the subsequent possibility to fulfill the plasmon resonance condition for several wavelengths and directions of vibration. When thickness is constant, as with flat layer deposition, only one resonance appears.

Another option, not depicted until now, is to produce what we call a double-sided DLUWT (Fig. 1, b). Once the layers are deposited, we turn around the taper and deposit on the other side. We can choose different materials or thicknesses for this second pair of layers, thus increasing the degrees of freedom for the design of the device, which is interesting for developing multianalyte sensors, but we have used the same thicknesses on both sides in this paper to provide a direct comparison with the other cases.

It is obvious that, to produce a fully symmetric deposit that covers the whole circumference of the section of the taper (Fig. 1, c), we must use some rotating mechanism inside the vacuum chamber. In some references, it is mentioned that the fiber is rotated in the chamber, but no technical explanation is given of the production process. Also, in many of the theoretical studies, fully cylindrical
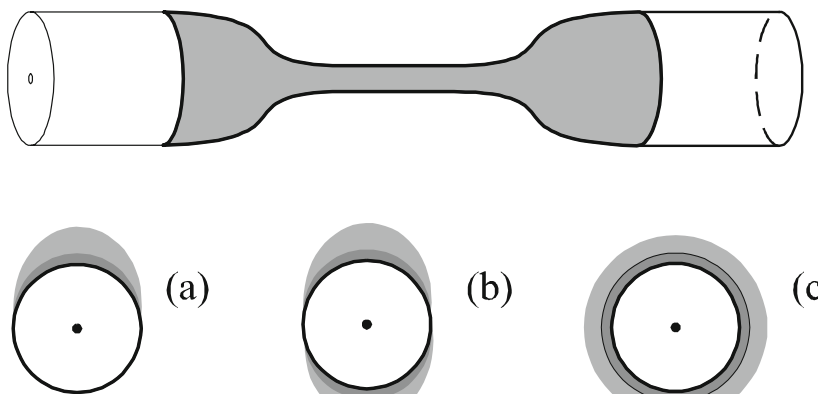

(a)

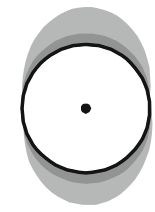

(b)

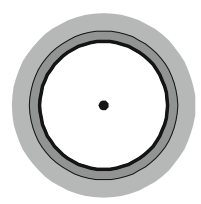

(c)

Fig. 1 Scheme of the different types of DLUWTs: $a$ asymmetrical, $b$ double-sided, $c$ symmetrical symmetry is simply assumed, without any suggestion about how it can be done in practice. In many of those cases, no explicit measurements of the real dependence on polarization are included, and no detailed mention of the polarization-controlling mechanisms needed to properly operate the sensor is made. Since we must deposit two different layers in the whole circumference of the taper (the second one in oxygen atmosphere) with due thickness accuracy and constancy, in the most fast and efficient way, it is important to develop a tool that permits us to make the deposits without opening the chamber. This implies that we must introduce equipment in the chamber, and this presents some limitations.

For instance, Obando et al. [25] note that motors capable of running in high-vacuum environments are expensive, and batteries must not be used due to chemical outgassing, so they propose to use the turntable that exists inside the chamber to place the samples, adding an element to attach the fibers. On the other hand, Dwivedi et al. [26] speak of a rotation of the fiber during the deposition, but they do not depict the setup that makes possible this rotation. Hu et al. [27] are the only ones, to our knowledge, to present a detailed scheme of a custom-made rotation mechanism they employ to deposit homogeneous $\mathrm{Pd}-\mathrm{Ag}$ alloy films on fibers, although no indications on the finally achieved independence on polarization or no mention to any polarization-controlling mechanisms are made, so we cannot assess up to what point they have reached their goal, since the constancy of the thickness will be experimentally demonstrated precisely by a full independence on polarization. As it is common in the literature, the authors use "TE" and "TM" to design polarizations in a completely arbitrary way, since no incidence plane is defined any more. Also, the deposition is only of one metallic layer. The authors specifically mention that deposition of a thin film on a 3D geometry such as a tapered fiber is much more technically demanding than depositing a thin film on a flat surface. They say that their setup is inexpensive, but they introduce the motor in the chamber and speak of a "custom-designed" electromotor with very restrictive specifications.

We present here a fabrication process to achieve the goal of controlled 3D deposition that only implies a very simple, inexpensive, and fully operative setup. The tool, made of Dural, has been designed and produced by us, and it is shown, both schematically and in a photograph, in Fig. 2. It is important to note that the motor and any electrical parts are placed outside the vacuum chamber, which is very advantageous. The movement is communicated to the piece inside the chamber by a rotating rod. This is then communicated to the parts supporting the fiber. The rotation speed can be controlled. A typical value of the speed of rotation is $25 \mathrm{rpm}$. 

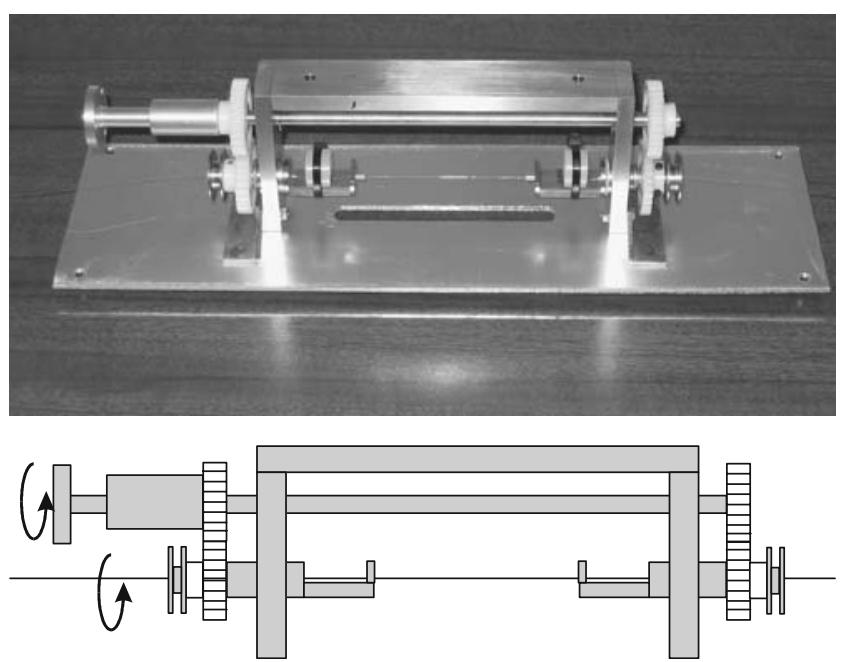

Fig. 2 Photograph and scheme of the element developed by us to provide a symmetrical deposition on the UWTs

With this procedure, we can deposit in a fast way, without opening the chamber and without affecting the deposition dynamics, two (or more) layers of the desired thickness, in a completely homogeneous way. The tapered fiber was single mode, reference F-SF of Fiber Optics, and presented this constructive parameters: taper waist, $35 \mu \mathrm{m}$; waist length, $6 \mathrm{~mm}$. We have used an aluminum 8 -nm-thick metal layer and a $\mathrm{TiO}_{2}$ dielectric layer, $60 \mathrm{~nm}$ thick. These values are in the range that we have used in the past for other DLUWT-based sensors, so we can compare their performance. We have developed in the past theoretical methods that model the behavior of the devices that permit us to select the thicknesses of the materials to have resonances in the desired ranges. Also, in the years that we have been working with DLUWT, we have optimized the deposition process, calibrating the times of deposition and characterizing the samples to permit an accurate control of the thicknesses employed. The appearance of the expected plasmon resonances for the right wavelengths is an a posteriori confirmation of the validity of the construction parameters.

The procedure to characterize the sensors is easy: in terms of the variation of refractive index, we expect a displacement of the plasmon dip with the variation of the refractive index of the outer medium. We vary it by a mixture of water and ethylene glycol. With respect to the polarization, we use a polarizer in the light source (an halogen lamp) and Lefebvre loops to change the state of polarization of the light incident on a straight, very short segment of fiber immediately before the transducing region (Fig. 3).

As the experimental results of the following paragraph prove, this fabrication procedure permits us to produce fully symmetrical deposits on DLUWTs and guarantee in this way that no polarization-controlling elements are needed for the development of field-measuring instruments.

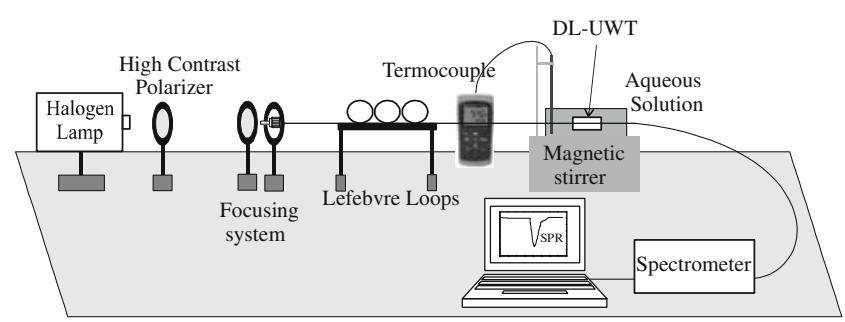

Fig. 3 Scheme of the experimental setup for the characterization of the transducers. The elements are submerged in a mixture of water and ethylene glycol, whose refractive index is varied in a known way. Polarization-controlling elements are included to evaluate the dependence of the response of the devices with polarization

\section{Experimental results}

The typical experimental behavior of symmetrical DLUWTs is shown in Fig. 4. There, we represent the normalized transmittance of the structure; "normalized" means the percentage of light transmitted when the transducer is submersed compared with the transmittance when the sensor is on air. Dashed and continuous lines are used to represent the use or nonuse of polarization-controlling elements. The spectral range shown is that of interest: we always take as reference the results obtained in the past with asymmetric devices, and, as we have said before, we have defined the parameters of the structure to achieve that a plasmon resonance appears in that range: the very clear experimental evidence, with a very well-defined resonance, shows that we have been successful in this point.

It is an important fact that not only $d o$ we have a resonance (which only appears for a given set of conditions and for a limited spectral range) but that we also have only one resonance, as it is the usual condition in flat-layerbased devices, but not in tapered-fiber-based devices. One

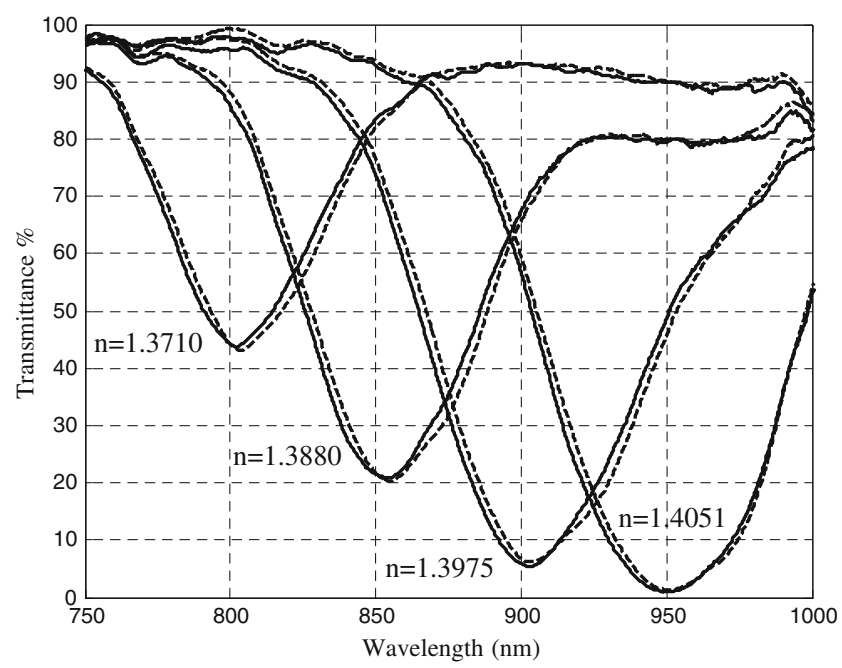

Fig. 4 Experimental behavior of symmetrical DLUWTs. A single wide plasmon dip appears in the spectral transmittance curve and the response shows no dependence on polarization 
of the major points of our work is to show how the multiple resonances appearing in asymmetrically deposited devices are due to the nonconstancy of the thickness and the subsequent possibility of simultaneously existing different resonance conditions for different wavelengths. That we have only one resonance in the case of symmetrical deposits is coherent with that reasoning: we recover the behavior of devices based on flat layers of constant thickness. Although, as it can be seen, the resonance is wide, which means that the thickness is not as constant as to provide a unique narrow resonance (as narrow as those of asymmetrically deposited devices). The small thickness gradient still remaining after the fabrication process with the rotating device can be made responsible for this width.

More important than this is the fact of the experimental evidence of the disappearance of any polarization dependence. The two curves corresponding to the use or nonuse of polarization-controlling elements are completely equivalent. This means that the main goal of the work is achieved; the fabrication process is successful, and the theoretical predictions are fulfilled.

A further confirmation of this is obtained when we consider the behavior of double-sided DLUWTs, as shown in Fig. 5 (again, dashed and solid lines mean polarization-controlling elements used and not used, respectively). The results are remarkable in themselves and also when compared to those of Fig. 4. Although one may think that these devices are near to the symmetrical ones, since a big part of the section is covered by the layers, we see that multiple resonances appear as in one-side asymmetrically deposited devices and that the (reduced) dependence on polarization is maintained. This can be explained easily by the fact that some directions of vibration of the field are privileged in terms of plasmon resonance, namely, those where the total thickness of the

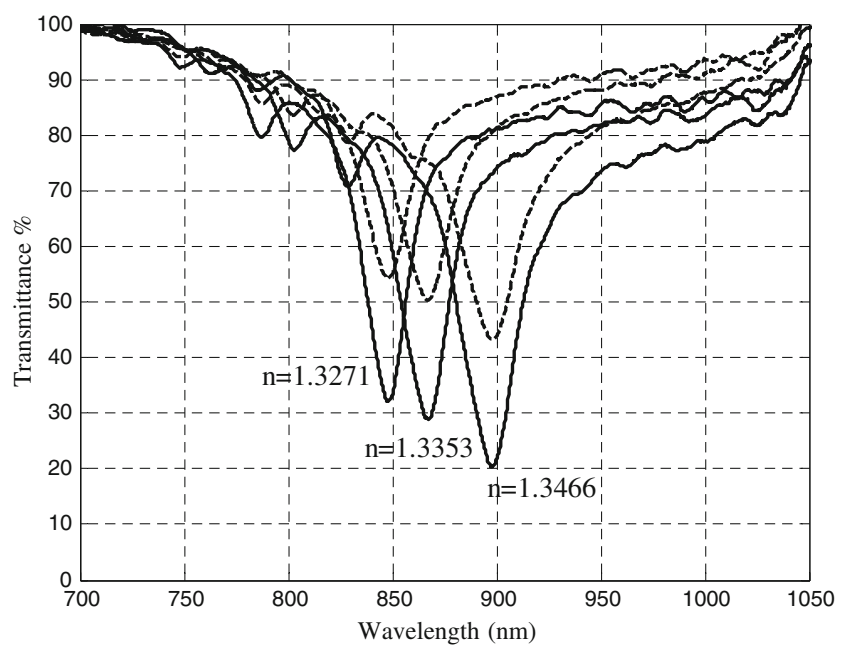

Fig. 5 Experimental behavior of double-sided DLUWTs. Multiple plasmon resonances appear, and the dependence on polarization has not completely disappeared

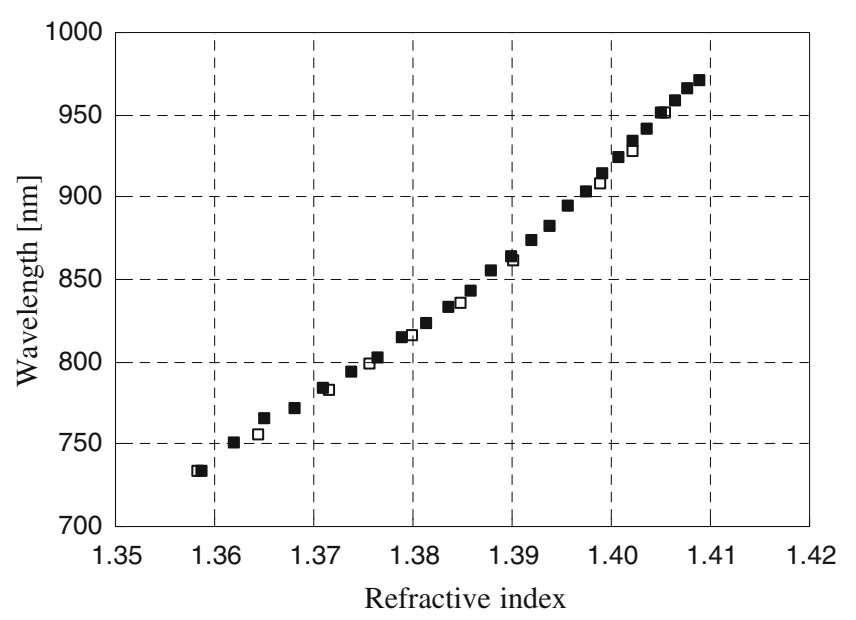

Fig. 6 Displacement of the plasmon resonance wavelengths of a symmetrical DLUWT with the variation (ascending, black squares; descending, white ones) of the refractive index of the surrounding medium

metal layer has been increased compared to that of one-side devices. The strong fluctuations of the thickness of the deposits when considering the whole circumference of the taper provide the possibility of simultaneous different resonance conditions that are fulfilled by several wavelengths, contrary to the case of symmetrically deposited devices, where these multiple plasmons "degenerate," so to speak into a single wider dip. These results show that a complete symmetry of the deposit is needed to achieve the desired goal of complete polarization dependence and corroborate the predictions of our theoretical modeling.

Finally, Fig. 6 shows that the new symmetrically deposited devices are very good refractometers. The experimental displacement of the resonance wavelength with the variation of the refractive index is presented. It can be seen that we have a good sensitivity of about $2.4 \times 10^{-4} \mathrm{RIU} / \mathrm{nm}$. Repeatability is also high, and no hysteresis effects appear.

This proved independence on polarization and good behavior as refractometers of symmetrical DLUWTs can be advantageously used in real measuring systems such as the one proposed in Fig. 7. The sensor is as simple as it can be, without any polarization-controlling elements. The reflective configuration permits us to reduce the size of the whole system to a few centimeters. The compactness, robustness, and small size are very important for any application.

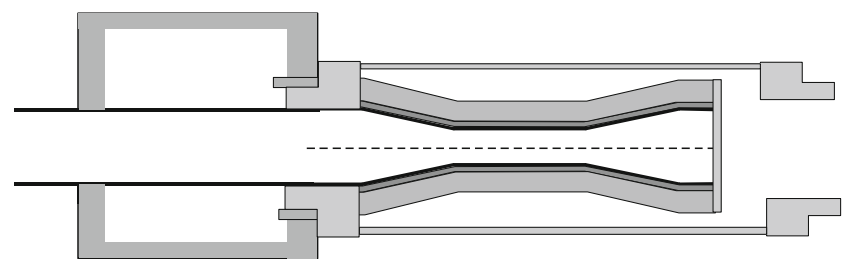

Fig. 7 A proposed scheme for a sensor based on a symmetrical DLUWT transducer in reflective configuration. The sensor is very small, compact, and robust 


\section{Conclusions}

In this work, we have shown how the polarization of the incident light influences in a decisive way the performance of any SPR sensor and how this dependence disappears when symmetrically, doubly deposited UWT optical fibers are used as transducers. This result, which is very interesting from the theoretical point of view, has very relevant consequences for the practical use of these devices as SPR sensors for chemical, biological, or environmental applications, since it permits us to use very simple and very compacts setups.

We have also presented a method for the fabrication of these devices, which provides a fully circular, constantthickness double deposit of layers on tapered fibers in a very efficient way. This method can be used for any other application that requires symmetrical deposits.

As our characterization shows, the symmetrical DLUWTs present only one wide plasmon resonance and a complete independence on polarization. This experimental result and the comparison with the behavior of the double-sided DLUWT confirm the theoretical predictions and improve our understanding of the dynamics of surface plasma waves in optical fibers. The measured displacements of the plasmon resonance wavelengths with the variation of the outer refractive index show that these elements can be used in any practical application as refractometers, presenting some remarkable advantages compared to the existing devices.

Acknowledgments This work has been partially supported by the Spanish Government research project NESTOR (Nuevas tecnologías de sensores de fibra óptica para la observación del medio marino), ref. CTM2004-03899, and Consejería de Educación de la Comunidad de Madrid research projects ROMA (Nueva generación de refractómetros de fibra óptica para aplicaciones medioambientales), ref. GR/AMB/ 0615/2004, and FUTURSEN ((Bio)sensores químicos avanzados para la medida in situ de la calidad de aguas basados en elementos específicos de reconocimiento y lectura multifuncional integrada), ref. S-0505/AMB-0374, and by Fondo Social Europeo and Fondo Europeo de Desarrollo Regional.

\section{References}

1. Homola J, Yee SS, Gauglitz G (1999) Surface plasmon resonance sensors: review. Sens Actuators B 54:3-15

2. Homola J (2003) Present and future of surface plasmon resonance biosensors. Anal Bioanal Chem 377:528-539

3. Raether H (1988) Surface plasmons on smooth and rough surfaces and on gratings. Springer, Berlin

4. Agranovich VM, Mills DL (eds) (1982) Surface polaritons. Electromagnetic waves at surfaces and interfaces. North-Holland, Amsterdam

5. Lin WB, Jaffrezic-Renault N, Gagnaire A, Gagnaire H (2000) The effect of polarization of the incident light-modeling and analysis of a SPR multimode optical fiber sensor. Sens Actuators B 84:198-204
6. Piliarik M, Homola J, Manikova Z, Ctyroky J (2003) Surface plasmon resonance sensor based on a single-mode polarizationmaintaining optical fiber. Sens Actuators B 90:236-242

7. Homola J, Yee SS (1998) Novel polarization control scheme for spectral surface plasmon resonance sensors. Sens Actuators B $51: 331-339$

8. Tseng S, Hsu K, Way H, Chen K (1997) Analysis and experiment of thin metal-clad fiber polarizer wave index overlay. IEEE Photonics Technol Lett 9:628-630

9. Johnstone W, Stewart G, Culshaw B, Hart T (1988) Fibre-optic polarisers and polarising couplers. Electron Lett 24(14):866-868

10. Díaz-Herrera N, Esteban Ó, Navarrete MC, Le Haitre M, González-Cano A (2006) In situ salinity measurements in seawater with a fibre-optic probe. Meas Sci Technol 17:22272232

11. Esteban Ó, Navarrete MC, González-Cano A, Bernabeu E (2000) Simple model of compound waveguide structures used as fiberoptic sensors. Opt Lasers Eng 33:219-230

12. Esteban Ó, Alonso R, Navarrete MC, González-Cano A (2002) Surface plasmon excitation in fiber optic sensors: a novel theoretical approach. J Lightwave Technol 20:448-453

13. Esteban Ó, Navarrete MC, González-Cano A (2005) Theoretical method for the study of plasmon generation in hybrid multilayeroptical fiber structures. IEEE Sens J 5(1):53-58

14. Lacroix S, Bourbonnais R, Gonthier F, Bures J (1986) Tapered monomode optical fibers: understanding large power transfer. Appl Opt 25:4421-4425

15. Birks TA, Li YW (1992) The shape of fiber tapers. J Lightwave Technol 10:432-438

16. Villatoro J, Monzón-Hernández D, Mejía E (2003) Fabrication and modelling of uniform-waist single-mode tapered optical fiber sensors. Appl Opt 42(13):2278-2283

17. Arregui FJ, Matías IR, López-Amo M (2000) Optical fiber strain gauge based on a tapered single-mode fiber. Sens Actuators A 79:90-96

18. González-Cano A, Bueno FJ, Esteban Ó, Díaz-Herrera N, Navarrete MC (2005) Multiple surface-plasmon resonance in uniform-waist tapered optical fibers with an asymmetric doublelayer deposition. Appl Opt 44(4):519-526

19. Monzón-Hernández D, Villatoro J (2006) High-resolution refractive index sensing by means of a multiple-peak surface plasmon resonance optical fiber sensor. Sens Actuators B 115:227-231

20. Díez A, Andrés MV, Cruz JL (1999) Hybrid surface plasma modes in circular metal-coated tapered fibers. J Opt Soc Am A $16: 2978-2982$

21. Díez A, Andrés MV, Culverhouse DO, Birks TA (1996) Cylindrical metal coated optical fibre devices for filters and sensors. Electron Lett 32:1390-1392

22. Díez A, Andrés MV, Cruz JL (2001) In-line fiber-optic sensors based on the excitation of surface plasma modes in circular metalcoated tapered fibers. Sens Actuators B 73:95-99

23. Bueno FJ, Esteban Ó, Díaz-Herrera N, Navarrete MC, GonzálezCano A (2004) Sensing properties of asymmetric double-layercovered tapered fibers. Appl Opt 43(8):1615-1620

24. Esteban Ó, Díaz-Herrera N, Navarrete MC, González-Cano A (2006) SPR sensors based on uniform-waist tapered fibers in reflective configuration. Appl Opt 45(28):7294-7298

25. Obando LA, Gentleman DJ, Holloway JR, Booksh KS (2004) Manufacture of robust surface plasmon resonance fiber optic based dip-probes. Sens Actuators B 100:439-449

26. Dwivedi YS, Sharma AK, Gupta BD (2008) Influence of design parameters on the performance of a surface plasmon sensor based fiber optic sensor. Plasmonics 3:79-86

27. Hu J, Jiang M, Lin Z (2005) Novel technology for depositing a $\mathrm{Pd}-\mathrm{Ag}$ alloy film on a tapered optical fibre for hydrogen sensing. J Opt A Pure Appl Opt 7:593-598 